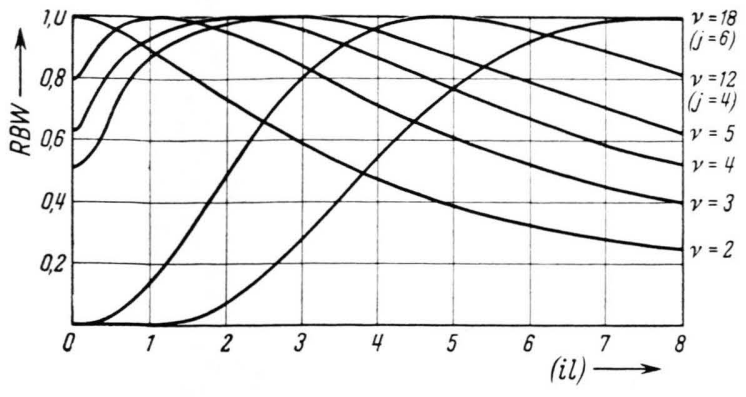

Abb. 2. RBW in Abhängigkeit vom linearen Energietransfer. Die auf gleichen biologischen Effekt bezogenen RBW-Werte gelten für den geradlinigen Anfangsteil der Dosiswirkungs. kurven. $v=$ kritische Anzahl der Ionenpaare; $i=$ Dichte der Primärionisationen längs der Teilchenbahn (Maß des LET) ; $l=$ mittlere Bahnlänge im Treffbereich je Teilchendurchgang. schweren Treffern praktisch ausschließlich längs derselben Teilchenbahn erfolgt. Die RBW-Werte für $v=2,3,4,5$ sind nach Gl. (10), für $v=12,18$ nach Gl. (7), (8) mit $j=4,6$, entsprechend einer mittleren Häufigkeit von 3 Ionenpaaren je Primärionisation berechnet worden. Das RBW-Maximum wurde in allen Fällen $=1$ gesetzt. Man entnimmt der Abb. 1, daß sich die Lage des RBW-Maximums für zunehmende Werte von $v$ immer weiter nach höheren Werten von $i l$ verschiebt. So liegt z. B. das Maximum für $v=4$ etwa bei $i l=2$, das Maximum für $v=5$ etwa bei $i l=3$, also bei einem relativ höheren Wert des linearen Energietransfers. Das Maximum selbst ist mit zunehmendem $v$ zunehmend deutlicher ausgeprägt.

\title{
NOTIZEN
}

\section{Photochemische Darstellung von Cyclopentadienylmangandicarbonyltolan}

Von W. Strohmeier und D. von Hobe

Institut für Physikalische Chemie der Universität Würzburg (Z. Naturforschg. 16 b, 402-403 [1961] ; eingegangen am 16. März 1961)

In Fortführung unserer Versuche zur photochemischen Darstellung von Derivaten der Metallcarbonyle ${ }^{1}$ untersuchten wir auch, ob $\pi$-Elektronendonatoren wie Acetylen und seine Derivate mit Metallcarbonylen oder deren Derivaten reagieren. Dabei zeigte sich, daß bei der UV-Bestrahlung einer Cyclohexanlösung von Cyclopentadienylmangantricarbonyl, der Tolan beigegeben war, Kohlenmonoxyd abgespalten wurde und sich Cyclopentadienylmangandicarbonyltolan

$$
\mathrm{C}_{5} \mathrm{H}_{5} \mathrm{Mn}(\mathrm{CO}){ }_{2} \mathrm{C}_{6} \mathrm{H}_{5} \mathrm{C}_{2} \mathrm{C}_{6} \mathrm{H}_{5}
$$

gebildet hatte, welches in Form einer braunen, luftbeständigen Substanz isoliert werden konnte. Die Verbindung ist in organischen Lösungsmitteln gut löslich; die Lösungen werden aber von Luft rasch zersetzt. Der Schmelzpunkt der Verbindung liegt bei $104-105^{\circ}$ (K of le r-Bank), der Sublimationspunkt im Hochvakuum bei $80-90^{\circ}$ (unter starker Zersetzung).

Bei der Aufarbeitung der Reaktionslösung wird in geringer Menge ein dunkelviolettes Produkt isoliert, dessen rotviolette Heptanlösung an der Luft wochenlang beständig ist. Diese Verbindung konnte noch nicht identifiziert werden. (Kohlenstoffgehalt: 89,7\% ; Schmp. $222^{\circ}$.)

1 W. Strohmeier u. Kl. Gerlach, Chem. Ber. 93, 2087 [1960]; Z. Naturforschg. 15 b, 413, 622, 675 [1960]; W. Sтrонmeier, G. Matthias u. D. von Hobe. Z. Naturforschg. 15 b. 813 [1960]; W. Strohmeier. Kl. Gerlach u. G. Matthias,
Darstellung vo n $\mathrm{C}_{5} \mathrm{H}_{5} \mathrm{Mn}(\mathrm{CO}){ }_{2} \mathrm{C}_{6} \mathrm{H}_{5} \mathrm{C}_{2} \mathrm{C}_{6} \mathrm{H}_{5}$ : $412 \mathrm{mg} \quad \mathrm{C}_{6} \mathrm{H}_{5} \mathrm{C}_{2} \mathrm{C}_{6} \mathrm{H}_{5} \quad(2,3 \mathrm{mMole})$ und $283 \mathrm{mg}$ $\mathrm{C}_{5} \mathrm{H}_{5} \mathrm{Mn}(\mathrm{CO})_{3}$ (1,38 mMole), gelöst in $25 \mathrm{~cm}^{3}$ Cyclohexan wurden mit der schon beschriebenen Versuchs. anordnung ${ }^{2}$ bis zur Abspaltung von 1,50 mMolen CO bestrahlt. Nach dem Abfiltrieren eines, bei der Reaktion ausgefallenen Produktes $(\sim 30 \mathrm{mg})$ wurde die Lösung an Aluminiumoxyd chromatographiert (GIULINI, Aktivitätsstufe II). Alle Operationen müssen unter Stickstoff ausgeführt werden, das $\mathrm{Al}_{2} \mathrm{O}_{3}$ wird im Hochvakuum entgast, dann mit $\mathrm{N}_{2}$ belegt und unter $\mathrm{N}_{2}$-Gas mit Cyclohexan eingeschwemmt. Zunächst wurde mit Petroläther eluiert. Es bildeten sich eine schneller laufende gelbe und eine langsam laufende violette Zone. Nachdem sich die Zonen getrennt hatten, eluierte man zur Erhöhung der Wanderungsgeschwindigkeit mit Petroläther-Benzol 1:1 die gelbe Zone und mit Benzol die violette Zone aus der Säule heraus. Nach dem Eindampfen des gelben Eluats im Vakuum blieb im Kolben ein schmieriger, brauner Rückstand. Der Kolben wurde an eine Hochvakuum-Apparatur angesetzt und bei $50^{\circ}$ Badtemperatur während $2 \mathrm{Stdn}$. das noch in geringer Menge vorhandene Tolan heraussublimiert. Zur Erhaltung eines analysenreinen Produktes mußte der nun festgewordene Kolbeninhalt in etwas Petroläther gelöst und unter peinlichstem Luftausschluß über eine Fritte filtriert werden. Der Kolben mit dem Filtrat wurde an die Hochvakuum-Apparatur angesetzt, das Lösungsmittel abgezogen und der feste Rückstand nochmals bei $45^{\circ}$ Badtemperatur 2 Stdn. im Hochvakuum erwärmt. Ausbeute: $165 \mathrm{mg}$ (34\%). Schmp. $104-105^{\circ}$ (K of . le r - Bank).

Z. Naturforschg. 15 b, 621 [1960]; W. Strohmeier, KL. Gerlach u. D. von Hobe, Chem. Ber. 94, 164 [1961].

2 W. Strohmeier u. Kl. Gerlach, Chem. Ber. 94, 398 [1961]. 
Analyse von $\mathrm{C}_{5} \mathrm{H}_{5} \mathrm{Mn}(\mathrm{CO}){ }_{2} \mathrm{C}_{6} \mathrm{H}_{5} \mathrm{C}_{2} \mathrm{C}_{6} \mathrm{H}_{5} \quad(354,3)$ :

Ber. C 71,19 H 4,27 Mn 15,5.

Gef. C 70,95 H 4,25 Mn 15,2.

Beim Eindampfen des rotvioletten Eluats im Vakuum wurden $15 \mathrm{mg}$ einer dunkelvioletten Substanz erhalten, deren Kohlenstoff-Gehalt bei $89,7 \%$ und deren Schmp. bei $222^{\circ}$ lag.

Wir danken der Deutschen Forschungsgemeinschaft und dem Fonds der Chemischen Industrie für die finan. zielle Unterstützung der vorliegenden Arbeit.

\section{Additionsverbindungen von Borhalogeniden mit Trialkylphosphinen}

\author{
Von Erhard Sirtl und Annemarie Adler \\ Forschungslaboratorium der Siemens \& Halske AG., \\ München \\ (Z. Naturforschg. 16 b, 403 [1961] ; eingegangen am 15. März 1961)
}

Bekanntlich weisen die 1 : 1-Additionsverbindungen des Trimethylamins mit verschiedenen Borverbindungen eine beachtliche Beständigkeit auf. So liegt z. B. der Schmp. von $\mathrm{BH}_{3} \cdot \mathrm{N}\left(\mathrm{CH}_{3}\right)_{3}{ }^{1}$ bei $94^{\circ}$, der von $\mathrm{BF}_{3} \cdot \mathrm{N}\left(\mathrm{CH}_{3}\right)_{3}{ }^{2}$ bei $150^{\circ}$ und der von $\mathrm{BCl}_{3} \cdot \mathrm{N}\left(\mathrm{CH}_{3}\right)_{3}{ }^{3}$ bei $244^{\circ}$. Unsere Untersuchungen an Bortrichlorid und -tribromid zeigten, daß auch Trialkylphosphine in der Lage sind, stabile Verbindungen gleichen Typs einzugehen.

So reagieren Bortrichlorid und Trimethylphosphin noch unterhalb $-78^{\circ}$ heftig unter Bildung einer feinkristallinen, weißen Masse, die durch Abpumpen der überschüssigen Komponente leicht in reinem Zustand erhalten werden kann. In etwas milderer Form - aber auch noch unterhalb $0^{\circ}$ - verlaufen die Umsetzungsreaktionen bei Bortrichlorid-Triäthylphosphin, Bortribromid-Trimethylphosphin und Bortribromid-Triäthylphosphin.

Sämtliche erhaltenen Verbindungen sind - im Gegensatz zu den Ausgangssubstanzen - recht beständig gegenüber Feuchtigkeit bzw. Sauerstoff. Sie sind in den meisten gebräuchlichen Lösungsmitteln nicht merklich löslich, lassen sich jedoch aus Aceton-Wasser gut umkristallisieren. Die Umsublimation bei vermin-

1 A. B. Burg u. H. J. Schlesinger, J. Amer. Chem. Soc. 59, 780 [1937].

2 A. B. Burg u. A. A. Green, J. Amer. Chem. Soc. 65, 1838 [1943].

3 E. Wiberg u. W. Sütterlin, Z. anorg. allg. Chem. 202, 31 [1931].

* Typische Analyse von $\mathrm{BCl}_{3} \cdot \mathrm{P}\left(\mathrm{CH}_{3}\right)_{3}$ : dertem Druck ist als weitere Reinigungsmethode zu nennen. Ein kurzes Aufschmelzen läßt die Präparate selbst an der Luft praktisch unverändert. Die Analyse der durch Umkristallisation oder Vakuum-Sublimation gereinigten Substanzen ergab in jedem Falle eine Bestätigung der Formel $\mathrm{BX}_{3} \cdot \mathrm{PR}_{3}\left(\mathrm{X}=\mathrm{Cl}, \mathrm{Br} ; R=\mathrm{CH}_{3}\right.$, $\left.\mathrm{C}_{2} \mathrm{H}_{5}\right)^{*}$.

Die meist nadelförmig ausgebildeten Kristalle scheinen nach D e b y e-S c h e r r e r-Aufnahmen und mikroskopischen Beobachtungen einer Klasse niedriger Symmetrie anzugehören. In der nachfolgenden Tabelle sind die Schmelzpunkte (nach Thi e le) der einzelnen Substanzen aufgeführt.

\begin{tabular}{|l|r|}
\hline & {$\left[{ }^{\circ} \mathrm{C}\right]$} \\
$\mathrm{BCl}_{3} \cdot \mathrm{P}\left(\mathrm{CH}_{3}\right)_{3}$ & 242 \\
$\mathrm{BBr}_{3} \cdot \mathrm{P}\left(\mathrm{CH}_{3}\right)_{3}$ & 256 \\
$\mathrm{BCl}_{3} \cdot \mathrm{P}\left(\mathrm{C}_{2} \mathrm{H}_{5}\right)_{3}$ & 71 \\
$\mathrm{BBr}_{3} \cdot \mathrm{P}\left(\mathrm{C}_{2} \mathrm{H}_{5}\right)_{3}$ & 110 \\
\hline
\end{tabular}

Tab. 1. Schmelzpunkt (nach Th i e l e) der einzelnen Substanzen.

Die verwendeten Borhalogenide wurden durch Umsetzung von Kaliumtetrafluoroborat mit Aluminiumchlorid bzw. -bromid erhalten und durch fraktionierte Kondensation auf Tensionsreinheit gebracht. Eine Grigna rd-Synthese nach HibBert ${ }^{4}$ führte $\mathrm{zu}$ den Trialkylphosphinen, die nach Long und SAckman ${ }^{5}$ über ihre Komplexverbindungen mit Silberjodid weiter gereinigt werden konnten. Die gemessenen Dampfdrucke des Trimethylphosphins stimmten vollkommen mit den Angaben in der Literatur ${ }^{6}$ überein.

$$
\begin{aligned}
& \text { Ber. B 5,6 P 16,0 C 18,6 H 4,7. } \\
& \text { Gef. B 5,3 P 15,6 C 19,1 H 4,8. }
\end{aligned}
$$

4 H. Hibbert, Ber. dtsch. chem. Ges. 39, 161 [1906].

5 L. H. Long u. J. F. Sackman, Trans. Faraday Soc. 53, 1606 [1957].

${ }^{6}$ E. J. Rosenbaum u. C. R. Sandberg, J. Amer. chem. Soc. 62 , 1622 [1940]. 